\title{
Simulação Numérica do Meio Reagente Ionizado Aplicando o Modelo de Equilíbrio Químico
}

A.P. SPILIMBERGO, C.J. AUTH, R. ISKHAKOVA, G. FELDMANN, Departamento de Física, Estatística e Matemática, Universidade Regional do Noroeste do Estado do Rio Grande do Sul - UNIJUÍ, Cx.P. 560, 98700-000 Ijuí, RS, Brasil.

\begin{abstract}
Resumo. Na modelagem matemática dos processos de combustão são largamente utilizados modelos de processos em equilíbrio químico que tradicionalmente são aplicados em meios reagentes com temperaturas variando no intervalo de 300 a $5000 K$. Freqüentemente existe a necessidade de determinar as características do meio reagente para temperaturas mais altas (até $20000 \mathrm{~K}$ ). Neste caso, os meios reagentes incluem frações consideráveis de elétrons e íons. No presente trabalho modifica-se o modelo matemático de equilíbrio químico e pesquisa-se as características do meio ionizado " $H+O$ " em amplo intervalo de temperatura (até $\approx$ $15000 \mathrm{~K}$ ) e pressão variando de 1 a $10 \mathrm{~atm}$. No modelo matemático, além das equações tradicionais (dissociação, conservação das espécies de átomos e de Dalton), é incluída também a equação da neutralidade da carga. Na base de dados as informações sobre a entalpia e a entropia das substâncias envolvidas, válidas para baixas temperaturas, foram alteradas para altas temperaturas, através de reaproximações.
\end{abstract}

\section{Introdução}

Na natureza e na indústria, seguidamente são encontrados meios ionizados gasosos, como produtos de combustão: em motores de combustão interna, em turbomotores, em motores de foguetes, em plasmas tecnológicos, etc. Freqüentemente é necessário conhecer as diferentes características do meio ionizado, como por exemplo a dependência entre as concentrações de íons (elétrons) e a temperatura. Estas informações podem ser obtidas com base em modelos matemáticos de equilíbrio químico (ver $[1,7]$ ) ou da cinética química (ver [6]).

No diagnóstico dos processos de combustão em motores de foguetes ou em motores de combustão interna são utilizados também detectores das características do plasma: se os processos ocorrem normalmente, estes detectores fixam o nível das concentrações, mas quando começam ocorrer algumas alterações em relação aos padrões normais, o nível das concentrações será distinto, e isto permitirá por exemplo, desligar o motor antes de sua destruição. Para que isto se realize com sucesso é necessário conhecer as propriedades (incluindo a composição) do meio ionizado. Um outro exemplo da importância da caracterização de meios ionizados é a aplicação 
de plasmas tecnológicos em processos de fabricação de dispositivos para indústria microeletrônica (ver [2]). Se o meio ionizado é "simples", a sua composição pode ser obtida facilmente através da Lei de Saha (ver [9]). Mas para meios "complexos" é necessário aplicar modelos que sejam invariantes, implatados nos softwares. Entre eles se destacam os modelos Gordon e McBride [5] e Alemassov et al. [1]. Gordon e McBride [5] utilizam o conceito de "potencial químico", que permite prever os meios neutros e ionizados através de um único código. Mas, neste software, além das equações de equilíbrio quimico são utilizadas também equações auxiliares, que aumentam o volume computacional. O modelo de Alemassov et al. [1] é baseado no conceito de "reações de dissociação" e apresenta menor volume de cálculo, mas para meios neutros e ionizados são utilizados softwares distintos, o que não é cômodo para os usuários. No presente trabalho são propostas modificações no modelo Alemassov et al. [1], que permitem (conservando o volume computacional) unificar o software envolvendo ambos os meios.

\section{Descrição e Alteração do Modelo Matemático}

O modelo Alemassov et al. [1] de equilíbrio químico dos meios neutros é composto pelas equações:

a) Dissociação das moléculas:

$$
\ln P_{j}-\sum_{i=1}^{n} a_{i j} \cdot \ln P_{i}+\ln K_{j}=0, \quad j=1, \ldots, m
$$

b) Conservação dos átomos:

$$
\ln \left(\sum_{j=1}^{m} a_{i j} \cdot P_{j}+P_{i}\right)-\ln M_{p}-\ln b_{i p}=0, \quad i=1, \ldots, n
$$

c) Dalton

$$
\ln \sum_{q=1}^{m+n} P_{q}-\ln P=0,
$$

onde $P_{i}, P_{j}$ e $P_{q}$ - pressões parciais do i-ésimo átomo, da j-ésima molécula/radical e da q-ésima substância dos produtos de combustão, respectivamente; $a_{i j}$ - quantidade do i-ésimo átomo na j-ésima molécula/radical; $K_{j}$ - constante de dissociação; $M_{p}$ - constante de proporcionalidade; $b_{i p}$ - quantidade do i-ésimo átomo na fórmula condicional do propelente. As constantes de dissociação das substâncias moleculares são determinadas por

$$
\ln K_{j}=\frac{\sum_{i=1}^{n} a_{i j} \cdot S_{i}^{o}-S_{j}^{o}}{R_{o}}-\frac{\sum_{i=1}^{n} a_{i j} \cdot H_{i}-H_{j}}{R_{o} T},
$$

sendo $R_{o}$ a constante universal dos gases; $S_{i}^{o}$ e $S_{j}^{o}$ a entropia do i-ésimo átomo e da j-ésima molécula/radical, respectivamente; $H_{i}$ e $H_{j}$ - entalpia molar do i-ésimo 
átomo e da j-ésima molécula/radical, respectivamente. Sendo a entropia e a entalpia calculadas através das expressões

$$
\begin{gathered}
H_{q}=A_{I_{q}}+\sum_{v=1}^{7} a_{v_{q}} \cdot x^{v}, \quad q=i, j ; \\
S_{q}=A_{S_{q}}+10^{-3} \cdot a_{1_{q}} \cdot \ln x+10^{-3} \sum_{v=2}^{3} \frac{v}{(v-1)} \cdot a_{v_{q}} \cdot x^{v-1}, \quad q=i, j,
\end{gathered}
$$

onde $A_{I}, A_{S}$ e $a_{v}$ são coeficientes para aproximação da entalpia e da entropia e $x=T / 1000$.

Para meios ionizados o sistema (2.1) - (2.3) é complementado por mais um tipo de equação - a equação da neutralidade da carga

$$
\sum_{k^{-}} P_{k^{-}}+P_{e}-\sum_{k^{+}} P_{k^{+}}=0
$$

onde $P_{k^{-}}, P_{e}$ e $P_{k^{+}}$são as pressões parciais dos íons negativos, elétrons e íons positivos respectivamente. Também nos grupos de equações (2.1) - (2.3) são incluídos termos com íons. Além disso no grupo de equações (2.1) aparecem novas equações sobre "dissociação" de íons. Por exemplo para um meio reagente simples: $H, H^{+}$, $H^{-}, e, H_{2}^{+}, H_{2}^{-}$e $H_{2}$, para a substância $H^{-}$será incluída no grupo (2.1) a equação:

$$
\frac{P_{H} \cdot P_{e}}{P_{H^{-}}}=K_{H^{-}}
$$

que, na forma logarítmica,

$$
\ln P_{H}+\ln P_{e}-\ln P_{H^{-}}=\ln K_{H^{-}}
$$

corresponde à equação de dissociação na sua forma geral (2.1) e corresponde, também, à reação de dissociação na base atômica

$$
H^{-}=H+e .
$$

Para o íon $H^{+}$esta concepção parece não servir, pois $H^{+}$é uma substância mais elementar do que $H$ e o íon $H^{+}$é gerado no resultado da decomposição do átomo $H$ pela reação

$$
H=H^{+}+e .
$$

Portanto, a equação de "dissociação" para o átomo $H$ é dada por

$$
\frac{P_{H^{+}} \cdot P_{e}}{P_{H}}=K_{H}
$$

sendo que sua forma logarítmica, 


$$
\ln P_{H^{+}}+\ln P_{e}-\ln P_{H}=\ln K_{H}
$$

não se submete a forma geral da Eq. (2.1). Surge então, o problema da formação da matriz dos coeficientes $a_{i j}$, onde um exemplo para o meio $H, H^{+}, H^{-}, e, O$, $\mathrm{O}^{+}, \mathrm{O}^{-}, \mathrm{O}_{2}, \mathrm{H}_{2}$ e $\mathrm{H}_{2} \mathrm{O}$ está apresentado na Tabela 1 , sendo que os coeficientes para as espécies $\mathrm{H}^{+}$e $\mathrm{O}^{+}$são indeterminados.

Uma possibilidade evidente de evitar essa discordância é introduzir, ao invés da base atômica, a base iônica $\left(H^{+}, \mathrm{O}^{+}, e\right)$ que está mostrada na Tabela 2. Mas neste caso (como foi mencionado acima) e necessário criar um código específico destinado somente para meios ionizados.

Tabela 1: Matriz dos coeficientes $a_{i j}$ na base atômica.

\begin{tabular}{|c|c|c|c|c|c|c|c|}
\hline & $\mathrm{H}_{2}$ & $\mathrm{O}_{2}$ & $\mathrm{H}_{2} \mathrm{O}$ & $\mathrm{H}^{-}$ & $\mathrm{O}^{-}$ & $\mathrm{H}^{+}$ & $\mathrm{O}^{+}$ \\
\hline$H$ & 2 & 0 & 2 & 1 & 0 & $?$ & 0 \\
\hline$O$ & 0 & 2 & 1 & 0 & 1 & 0 & $?$ \\
\hline$e$ & 0 & 0 & 0 & 1 & 1 & $?$ & $?$ \\
\hline
\end{tabular}

Tabela 2: Matriz dos coeficientes $a_{i j}$ na base iônica.

\begin{tabular}{|c|c|c|c|c|c|c|c|}
\hline & $\mathrm{H}_{2}$ & $\mathrm{O}_{2}$ & $\mathrm{H}_{2} \mathrm{O}$ & $\mathrm{H}^{-}$ & $\mathrm{O}^{-}$ & $\mathrm{H}$ & $\mathrm{O}$ \\
\hline $\mathrm{H}^{+}$ & 2 & 0 & 2 & 1 & 0 & 1 & 0 \\
\hline $\mathrm{O}^{+}$ & 0 & 2 & 1 & 0 & 1 & 0 & 1 \\
\hline$e$ & 2 & 2 & 3 & 2 & 2 & 1 & 1 \\
\hline
\end{tabular}

Então, seria preferível buscar uma nova possibilidade de modelar os meios ionizados dentro da abordagem da base atômica. Para isso, a Eq. (2.6) é reescrita como

$$
\ln P_{H}-\ln P_{e}-\ln P_{H^{+}}=-\ln K_{H},
$$

com

$$
-\ln K_{H}=\frac{S_{H}-S_{e}-S_{H^{+}}}{R_{o}}-\frac{H_{H}-H_{e}-H_{H^{+}}}{R_{o} T} .
$$

Observa-se que (2.7) continua não estando na forma geral (2.1). Substituindo-se, então, (2.8) em (2.7), obtém-se

$$
\ln P_{H}-\ln P_{e}-\ln P_{H^{+}}=\frac{S_{H}-S_{e}-S_{H^{+}}}{R_{o}}-\frac{H_{H}-H_{e}-H_{H^{+}}}{R_{o} T} .
$$

$\mathrm{Na}$ forma geral (2.1), considerando a base atômica, estas equações são escritas como

$$
\begin{gathered}
a_{\left(H H^{+}\right)} \ln P_{H}+a_{\left(e H^{+}\right)} \ln P_{e}-\ln P_{H^{+}}=\ln K_{H^{+}} \\
\ln K_{H^{+}}=\frac{a_{\left(H H^{+}\right)} S_{H}+a_{\left(e H^{+}\right)} S_{e}-S_{H^{+}}}{R_{o}}-\frac{a_{\left(H H^{+}\right)} H_{H}+a_{\left(e H^{+}\right)} H_{e}-H_{H^{+}}}{R_{o} T}
\end{gathered}
$$

onde substituindo-se (2.11) em (2.10) obtém-se: 


$$
\begin{aligned}
& a_{\left(H H^{+}\right)} l n P_{H}+a_{\left(e H^{+}\right)} l n P_{e}-\ln P_{H^{+}}= \\
& \quad \frac{a_{\left(H H^{+}\right)} S_{H}+a_{\left(e H^{+}\right)} S_{e}-S_{H^{+}}}{R_{o}}-\frac{a_{\left(H H^{+}\right)} H_{H}+a_{\left(e H^{+}\right)} H_{e}-H_{H^{+}}}{R_{o} T} .
\end{aligned}
$$

Comparando (2.9) com (2.12), facilmente se conclui que ambas as equações serão coincidentes se $a_{\left(H H^{+}\right)}=1$ e $a_{\left(e H^{+}\right)}=-1$. Este último valor não tem sentido físico, mas pode ser entendido como a falta de elétron no íon $H^{+}$, e se considera sua presença com o coeficiente "-1". Portanto, dessa forma, é possível modelar os meios ionizados conservando a base atômica e as reações básicas e praticamente sem alterações no algoritmo e aplicativo ${ }^{[8]}$ dos meios neutros. A matriz $a_{i j}$ completa está apresentada na Tabela 3.

Tabela 3: Matriz dos coeficientes $a_{i j}$ na base atômica.

\begin{tabular}{|c|c|c|c|c|c|c|c|}
\hline & $\mathrm{H}_{2}$ & $\mathrm{O}_{2}$ & $\mathrm{H}_{2} \mathrm{O}$ & $\mathrm{H}^{-}$ & $\mathrm{O}^{-}$ & $H^{+}$ & $\mathrm{O}^{+}$ \\
\hline$H$ & 2 & 0 & 2 & 1 & 0 & 1 & 0 \\
\hline$O$ & 0 & 2 & 1 & 0 & 1 & 0 & 1 \\
\hline$e$ & 0 & 0 & 0 & 1 & 1 & -1 & -1 \\
\hline
\end{tabular}

As equações de conservação dos átomos não se alteram (nenhum coeficiente $a_{i j}$ com sinal "-" é incluído na Eq. (2.2)), além disso a equação da neutralidade da carga está submetida a forma geral (2.2), tornando-se a equação da conservação do elétron

$$
\sum_{j=1}^{m} a_{e j} P_{j}+P_{e}=M_{p} \cdot b_{e p}=0,
$$

onde $a_{e j}=0$ para as substâncias neutras; $a_{e j}=1$ para íons negativos; $a_{e j}=-1$ para íons positivos; $b_{e p}=0$, pois a carga elétrica do propelente é zero. Assim a forma logarítmica para a Eq. (2.13) não pode ser usada e somente este fato condiciona as alterações no algoritmo e no aplicativo.

\section{Pesquisas Numéricas}

Os atuais aplicativos (SP-273 ${ }^{[5]}$, ADTIJUI ${ }^{[8]}$, etc.) utilizam bases de dados sobre as substâncias (TERMO ${ }^{[5]}$, BURCAT ${ }^{[3]}$, TTU $^{[1]}$ ) com temperaturas variando de 300 até $6000 \mathrm{~K}$. Devido a isso, inicialmente foi necessário obter informações sobre as propriedades termodinâmicas $\left(H_{q}\right.$ e $\left.S_{q}\right)$ para temperaturas de até $20000 \mathrm{~K}$ que foram encontradas, em forma tabelada, em Glusko et al. [4]. Para adaptá-las ao software ADTIJUI, foi necessário interpolar as dependências $H_{q}, S_{q}=f(T)$ na forma dos polinômios (2.4) e (2.5), criando uma nova base de dados. A interpolação foi realizada utilizando polinômios de Chebyshev. Os erros máximos nos diferentes intervalos de temperatura, estão mostrados na Tabela 4. Nesta tabela, os $\delta_{q}(K)$ significam os erros na temperatura do plasma, no caso do uso do polinômio $H_{q}=$ $f(T)$ ao invés da forma apresentada em Glusko et al. [4]. Como é visível, os erros são 
pequenos e são admissíveis para demandas tanto de pesquisa como da prática. Além disso observou-se também que os erros mais notáveis ocorreram para as espécies trimoleculares $\left(\mathrm{H}_{2} \mathrm{O}\right.$ e $\left.\mathrm{CO}_{2}\right)$. Na Fig. 1 está apresentada a comparação dos resultados obtidos pelo software ADTIJUI com os dados observados em Vargaftik [10] para o plasma de argônio com pressões de 1 e $0,1 \mathrm{~atm}$.

Tabela 4: Erros máximos entre valores tabelados e interpolados da entalpia $\left(H_{q}\right)$.

\begin{tabular}{|c|c|c|c|c|c|c|c|}
\hline$\Delta T(K) \rightarrow$ & $\begin{array}{c}1000 \\
\text { até } \\
\delta_{\text {max }}(\mathrm{K}) \downarrow\end{array}$ & $\begin{array}{c}3000 \\
\text { até } \\
5000\end{array}$ & $\begin{array}{c}5000 \\
\text { até } \\
8000\end{array}$ & $\begin{array}{c}8000 \\
\text { até } \\
11000\end{array}$ & $\begin{array}{c}11000 \\
\text { até } \\
14000\end{array}$ & $\begin{array}{c}14000 \\
\text { até } \\
17000\end{array}$ & $\begin{array}{c}17000 \\
\text { até } \\
20000\end{array}$ \\
\hline$\delta_{H}$ & 0,033 & 0,025 & 0,055 & 0,052 & 0,060 & 0,039 & 0,047 \\
\hline$\delta_{O}$ & 1,625 & 1,626 & 1,715 & 0,610 & 0,337 & 0,246 & 0,191 \\
\hline$\delta_{H_{2} O}$ & 10,278 & 9,089 & 9,377 & 5,644 & 4,555 & 3,337 & 3,905 \\
\hline$\delta_{O H}$ & 4,983 & 4,719 & 4,234 & 2,587 & 2,145 & 1,676 & 2,199 \\
\hline$\delta_{O_{2}}$ & 4,499 & 3,877 & 13,314 & 5,216 & 3,896 & 3,636 & 5,310 \\
\hline$\delta_{H_{2}}$ & 5,474 & 4,839 & 5,389 & 3,524 & 3,021 & 2,142 & 2,368 \\
\hline
\end{tabular}

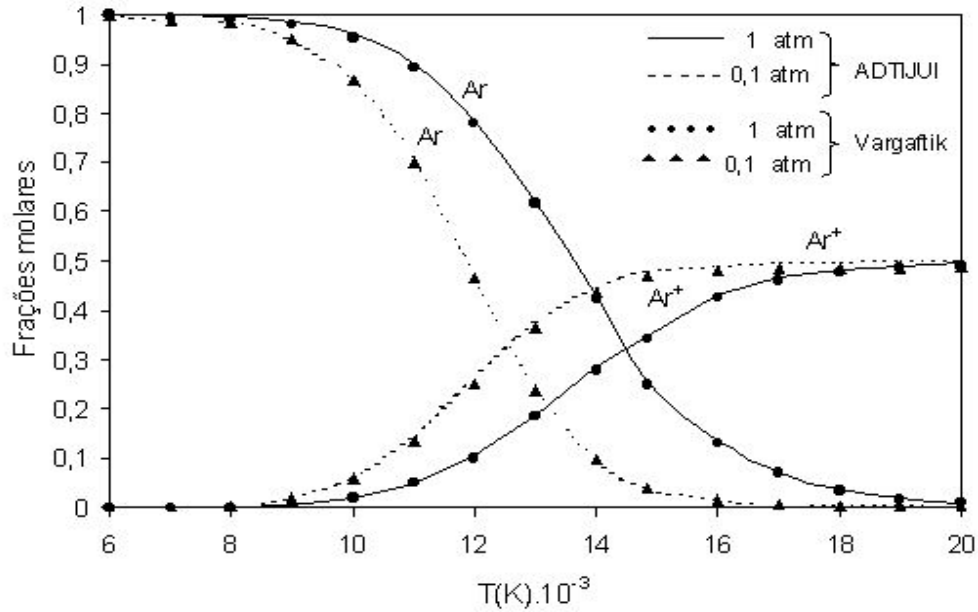

Figura 1: Comparação das frações molares do $A r$ e do $A r^{+}$

As simulações numéricas foram realizadas para o meio ionizado " $H+O$ ", em condições estequiométricas, nos intervalos $T=1000 \ldots 15000 \mathrm{~K}$ e $P=1 \ldots 10 \mathrm{~atm}$, levando em conta as substâncias $\mathrm{O}, \mathrm{H}, \mathrm{O}_{2}, \mathrm{H}_{2}, \mathrm{OH}, \mathrm{H}_{2} \mathrm{O}, e, \mathrm{O}^{+}, \mathrm{O}^{-}, \mathrm{H}^{+}, \mathrm{H}^{-}$, $\mathrm{O}_{2}^{+}, \mathrm{O}_{2}^{-}, \mathrm{OH}^{+}, \mathrm{OH}^{-}, \mathrm{H}_{2} \mathrm{O}^{+}, \mathrm{H}_{3} \mathrm{O}^{+}$e os resultados estão apresentados nas Figuras $2-5$.

$\mathrm{Na}$ Figura 2 estão mostradas as alterações das substâncias $\mathrm{H}_{2} \mathrm{O}, \mathrm{H}_{2}$ e $\mathrm{O}_{2}$ em função de $T$ para pressões de $1 \mathrm{~atm}$ e $10 \mathrm{~atm}$. Para $T=1000 \mathrm{~K}$, o meio reagente contém somente $\mathrm{H}_{2} \mathrm{O}$. Mas, a partir de $2000 \mathrm{~K}(P=1 \mathrm{~atm})$, a água começa a 


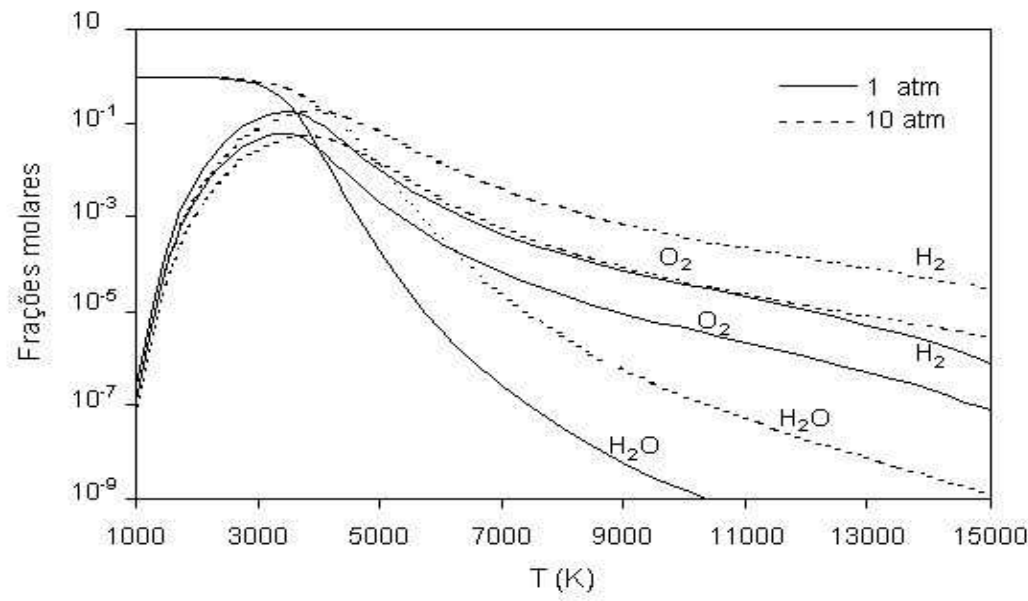

Figura 2: Alterações das frações molares de $\mathrm{H}_{2} \mathrm{O}, \mathrm{O}_{2}$ e $\mathrm{H}_{2}$ em função da $\mathrm{T}$

dissociar-se em $\mathrm{H}_{2}, \mathrm{O}_{2}, \mathrm{O}, \mathrm{H}$ e $\mathrm{OH}$ e praticamente desaparece para $\mathrm{T} \approx 4500 \mathrm{~K}$. As substâncias $\mathrm{H}_{2}, \mathrm{O}_{2}$ e $\mathrm{OH}$ apresentam suas concentrações máximas para $\mathrm{T} \approx 3500$ $\mathrm{K}$, pois sendo elas os produtos da decomposição da água, também começam a dissociar-se em átomos para $T>3500 \mathrm{~K}$. Para temperaturas maiores que 6500 $\mathrm{K}$ estas substâncias apresentam concentrações pequenas (mas não desaparecendo totalmente).

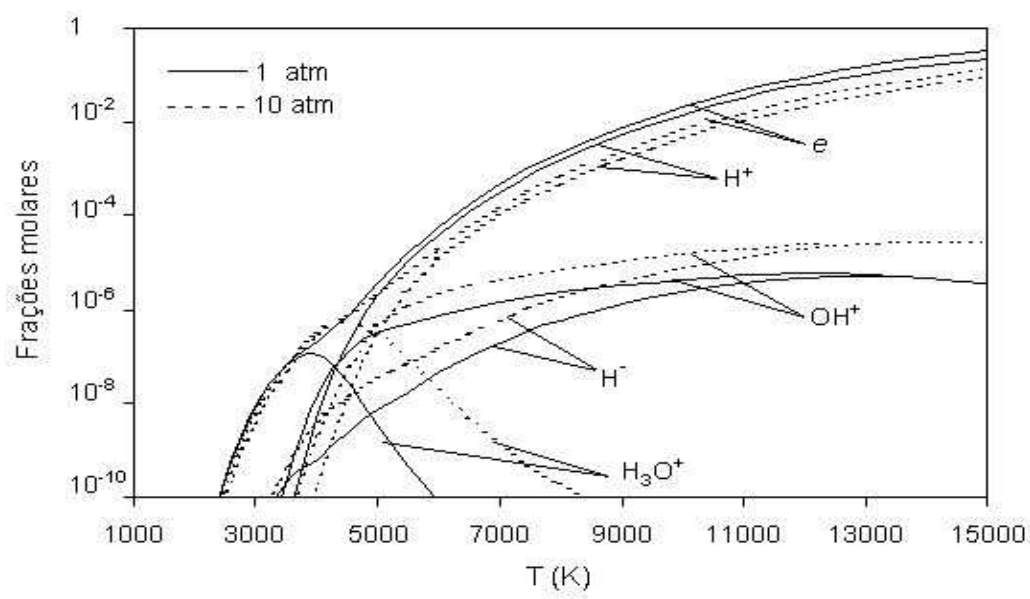

Figura 3: Alterações das espécies $\mathrm{H}_{3} \mathrm{O}^{+}, e, \mathrm{OH}^{+}, \mathrm{H}^{+}$e $\mathrm{H}^{-}$em função da $\mathrm{T}$

Com o aumento da temperatura começam a ser gerados íons e elétrons - Figuras 3 e 4. Entre os íons considerados no plasma, as concentrações de $\mathrm{O}_{2}^{-}$e $\mathrm{OH}^{-}$são pequenas $\left(r_{\mathrm{OH}^{-}}, r_{\mathrm{O}_{2}^{-}}<10^{-10}\right.$ em todo intervalo da temperatura). Os íons $\mathrm{H}_{2} \mathrm{O}^{+}$ 
e $\mathrm{H}_{3} \mathrm{O}^{+}$também possuem concentrações pequenas com máximos: $r_{\mathrm{H}_{2} \mathrm{O}^{+}}=5.10^{-8}$ $(T=4500 \mathrm{~K})$ e $r_{H_{3} O^{+}}=10^{-7}(T=4000 \mathrm{~K})$. Observa-se a importância do íon $\mathrm{H}_{3} \mathrm{O}^{+}$ na formação dos elétrons para baixas temperaturas. Já os íons $\mathrm{O}^{-}, \mathrm{O}_{2}^{+}, \mathrm{H}^{-}$e $\mathrm{OH}^{+}$ são bem mais estáveis. Eles começam a ser formados quando $T \approx 3000 \mathrm{~K}$ atingindo estabilidade em suas concentrações $\left(r_{O^{-}} \approx 10^{-6} ; r_{O_{2}^{+}} \approx 10^{-6} ; r_{O H^{+}} \approx 10^{-5} \mathrm{e}\right.$ $r_{H^{+}} \approx 10^{-6}$ ) para $T \approx 7000 \mathrm{~K}$. Observa-se que $O^{-}$e $H^{-}$são os únicos íons negativos, Fig. (3) e (4), formados em frações notáveis no meio ionizado. Os íons restantes são positivos e são responsáveis pela formação dos elétrons. Com fração $r_{e} \approx 10^{-10}$ os elétrons começam a surgirem para $T \approx 2500 \mathrm{~K}$, principalmente devido ao íon $\mathrm{H}_{3} \mathrm{O}^{+}$(até $T \approx 3500 \mathrm{~K}$ ). Após, a fração $r_{e}$ continua aumentando no intervalo de 3500 até $5000 \mathrm{~K}$, devido a formação dos íons: $\mathrm{H}_{3} \mathrm{O}^{+}, \mathrm{OH}^{+}, \mathrm{O}_{2}^{+}, \mathrm{H}^{+}$e $\mathrm{O}^{+}$. Mas a partir de $T=5500 \mathrm{~K}, r_{e}$ cresce somente devido aos íons $\mathrm{O}^{+}$e $H^{+}$, o que continua até $T=15000 \mathrm{~K}$. Observa-se que nessa temperatura o meio reagente já se encontra consideravelmente ionizado: $r_{e} \approx r_{O^{+}}+r_{H^{+}} \approx 0,31 ; r_{O} \approx 0,12$ e $r_{H} \approx 0,25$ para $P=1 \mathrm{~atm}$.

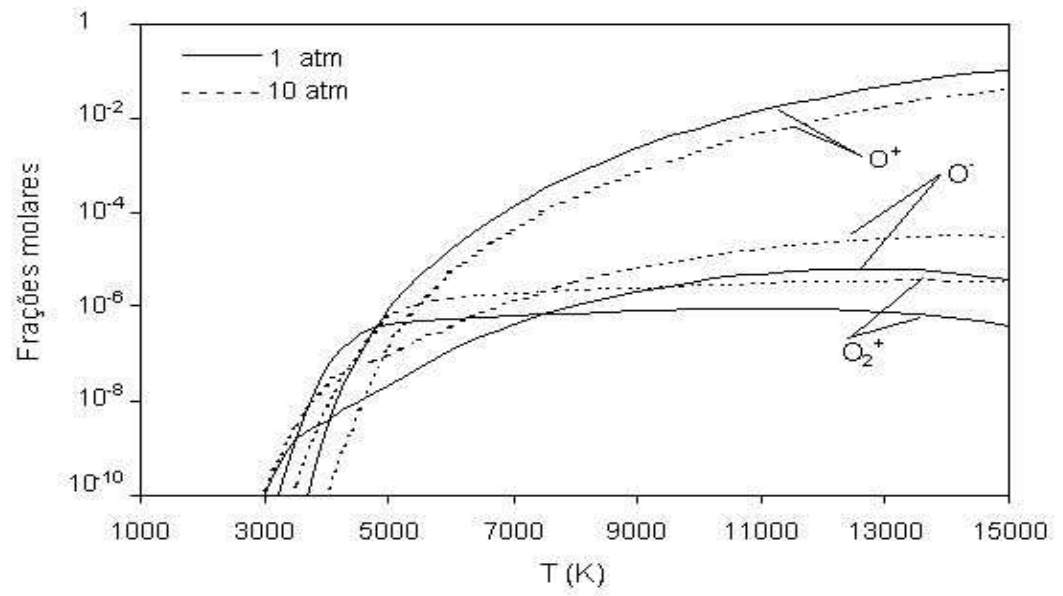

Figura 4: Alterações das concentrações de $O^{+}, O^{-}$e $O_{2}^{+}$em função da $T$

Para a pressão de 10 atm as transformações do meio reagente são análogas, mas com retardamento da dissociação. Por exemplo, $r_{e}$ no fim do intervalo considerado da temperatura, diminui mais do que 2 vezes na passagem de $P=1$ até $P=10$ atm.

Na Figura 5 estão mostradas as alterações das propriedades do meio reagente: $\mu$ - massa molecular média; $C_{p f}$ e $C_{p e}$ - calores específicos "congelado" e equilibrado. É visível que: $\mu$ sofre uma brusca alteração no intervalo de 2500 a $4500 \mathrm{~K}$, logo após torna-se praticamente constante até $9000 \mathrm{~K}$, voltando a partir daí novamente a diminuir; $C_{p f}$ é praticamente constante em todo intervalo da temperatura; o valor de $C_{p e}$ é o mesmo de $C_{p f}$ quando $T=1000 \ldots 2000 \mathrm{~K}$; no intervalo de 2000 a $5500 \mathrm{~K}$ apresenta um máximo; quando $T=5500 \ldots 9000 \mathrm{~K}$ apresenta pequenas alterações e a partir de $9000 \mathrm{~K}$ vizualiza-se novamente o seu crescimento; todos esses efeitos são provocados pelas alterações no meio reagente. A queda brusca da massa molecular 


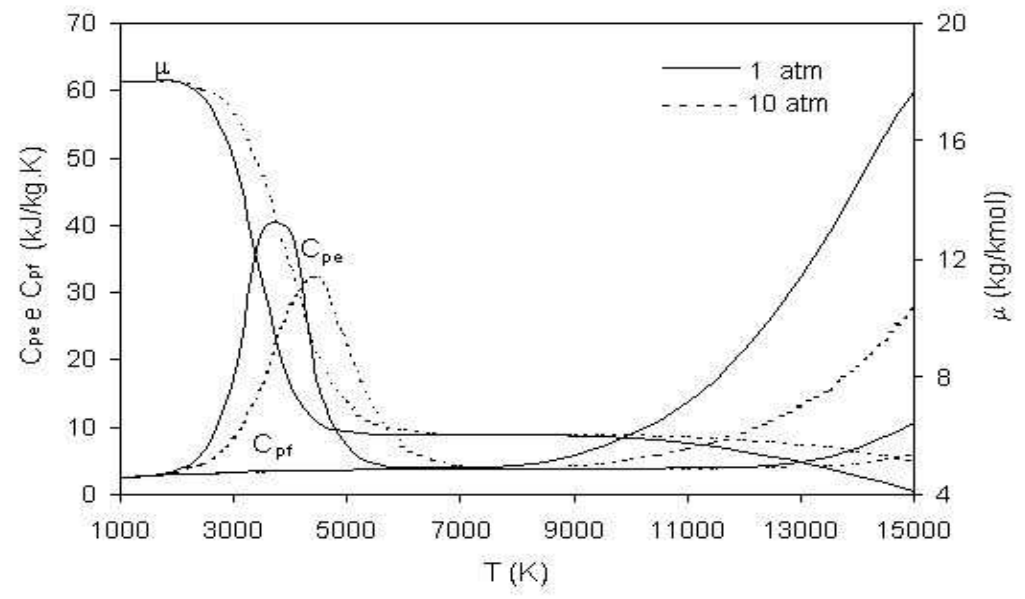

Figura 5: Alterações de $C_{p e}, C_{p f}$ e $\mu$ em função da $T$

(de 18 até $6 \mathrm{~kg} / \mathrm{Kmol}$ ) é conseqüência da dissociação da água, um "patamar" (para $T=4500 \ldots 9000 \mathrm{~K}$ ) é condicionado pela inalterabilidade da composição, e a queda final pela formação intensiva dos elétrons. Pela expressão (ver [1]) que fornece o valor de $C_{p e}$ :

$$
C_{p e}=C_{p f}+\frac{1}{M_{p} \cdot T} \cdot\left[P \cdot \sum_{q} r_{q} \cdot H_{q} \cdot\left(\frac{\partial \ln r_{q}}{\partial \ln T}\right)_{p}\right]-\frac{1}{T} \cdot\left(\frac{\partial \ln M_{p}}{\partial \ln T}\right)_{p}
$$

é fácil concluir que quando os gradientes $d r_{q} / d T$ são bastante altos (em módulo), o valor de $C_{p e}$ cresce e seu máximo, no intervalo de 2000 a $5500 \mathrm{~K}$, é provocado pelos altos valores dos gradientes: $d r_{q} / d T$ onde $q=O, H, H_{2} O$. Para $T>9000 \mathrm{~K}$, o aumento de $C_{p e}$ é provocado por $d r_{q} / d T \operatorname{com} q=e, O^{+}, O, H^{+}, H$.

\section{Conclusões}

1. Foi modificado o modelo de cálculo de sistemas quimicamente equilibrados (Alemassov et al. [1]), a saber: introdução de coeficientes estequiométricos negativos; transformação da equação da neutralidade da carga em uma equação de conservação de átomos; criação de uma nova base de dados com $T=1000 \ldots 20000 \mathrm{~K}^{[4]}$. No resultado, o software ADTIJUI ${ }^{[8]}$, para prever as características do meios reagentes neutros, foi adaptado para meios reagentes ionizados evitando assim o uso de um código específico para esse tipo de meio.

2. Os primeiros cálculos foram realizados, em condições estequiométricas, para o meio "H+O", com $P=1 \ldots 10$ atm e $T=1000 \ldots 15000 \mathrm{~K}$. Simulações mostraram que até $T=4500 \mathrm{~K}, \mathrm{H}_{2} \mathrm{O}$ dissocia-se em $\mathrm{H}_{2}, \mathrm{O}_{2}, O H, O$ e $H$; até $T=6500 \mathrm{~K}$, $\mathrm{H}_{2}$ e $\mathrm{O}_{2}$ dissociam-se em $\mathrm{O}$ e $\mathrm{H}$; os íons começam a serem formados a partir de $T=2500 \mathrm{~K}$; os elétrons são formados inicialmente por conta do íon $\mathrm{H}_{3} \mathrm{O}^{+}$; no intervalo $3500<\mathrm{T}<5500 \mathrm{~K}$ por conta dos íons $\mathrm{H}_{3} \mathrm{O}^{+}, \mathrm{OH}^{+}, \mathrm{O}_{2}^{+}, \mathrm{O}^{+}$e $\mathrm{H}^{+}$e, para $T>5500 \mathrm{~K}$, por conta de $H^{+}$e $O^{+}$. 
3. Em relação às propriedades dos produtos de combustão analisadas pode-se observar que $\mu$ sofre uma brusca alteração no intervalo de 2500 a $4500 \mathrm{~K}$, como consequiência da dissociação da água, logo após mantém-se praticamente constante até $T=9000 \mathrm{~K}$, onde volta a diminuir; $C_{p f}$ mantém-se praticamente constante em todo intervalo de variação da temperatura; $C_{p e}$ apresenta um máximo para $T=2000 \ldots 5500 \mathrm{~K}$ e um crescimento para $T>9000 \mathrm{~K}$ (provavelmente com a formação de mais um máximo para $T>15000 \mathrm{~K}$ ), efeitos estes provocados pelas alterações no meio reagente.

\begin{abstract}
The mathematical models of combustion processes are generally based on chemical equilibrium models that are valid at low temperatures $(300 \ldots 5000 \mathrm{~K})$. For the analysis of ionized media at higher temperatures, the number fraction of ions and electrons has to be taken in account. In the present present paper is modified the mathematical model of chemical equilibrium and is performed a numerical simulation of the simple "H+O" ionized medium in a wider range of temperatures (up to $15000 \mathrm{~K}$ ), and pressure varying from 1 to $10 \mathrm{~atm}$. In the mathematical model we use the traditional set of equations (dissociation, conservation of atoms and Dalton), added to the charge-neutrality equation. The database of enthalpies and entropies of the involved species valid at low temperatures were expanded for high temperatures by reaproximation.
\end{abstract}

\title{
Referências
}

[1] V.S. Alemassov, A.F. Dregalin e A.P. Tishin, "Propriedades Termodinâmicas e Termofísicas dos Produtos de Combustão", Vol. 1, VINITI, Moscou, 1971.

[2] M.I. Boulos, P. Fauchais e E. Pfender, "Thermal Plasmas", Vol. 1, Plenum Press, New York, 1994.

[3] A. Burcat, "Third Millennium Ideal Gas and Condensed Phase Thermochemical Database for Combustion", Technion Aerospace Engineering (TAE), Report $N^{o}$ 867, New York, 2001.

[4] V.P. Glushko et al, "Guia das Propriedades Termodinâmicas e Termofísicas de Substâncias Individuais", Nauka, Moscou, 1982.

[5] S. Gordon e B.J. McBride, "NASA SP273 - Computer Program for Calculation of Complex Chemical Equilibrium Compositions, Rocket Performance, Incident and Reflected Schoks, and Chapman-Jouguet Detonations", Washington, 1971.

[6] R.J. Kee, F.M. Rupley e J.A. Miller, "CHEMKIN-II: A Fortran Chemical Kinetics Package for the Analysis of Gas Phase Chemical Kinetics", Supersedes SAND89-8009, UA, 1989.

[7] B.J. McBride, S. Gordon e M.A. Reno, "Coefficients for Calculating Thermodynamic and Transport Properties of Individual Species", NASA, Technical Memorandum 4513, Washington, 1993.

[8] A.P. Spilimbergo, R.L. Ishkakova e C.A. Castelli, Modelado de flujos quimicamente reactivos em toberas, Informacion Tecnologica, 12 (2001), 135-142.

[9] R. Thoukvatoulline e G. Feldmann, Effect of pulsation on the determination of temperature with the method of absolute line intensity, J. Phys. D: Appl. Phys., 33 (2000), 2420-2424.

[10] N.B. Vargaftik, "Guia das Propriedades Termofísicas de Gases e Líquidos", Ciência, Moscou, 1972. 